\title{
AVALIAÇÃO DE RESÍDUOS DE AGROTÓXICOS EM POLPAS DE MORANGO INDUSTRIALIZADAS
}

\author{
VANESSA HELOISA FERREIRA DE FARIA * \\ BÁRBARA MESQUITADIAS ** \\ MÁRCIA CASSIMIRAMARCOS DA COSTA *** \\ VIRGINIA RIBEIRO DA SILVA **** \\ ANELISE LAPERTOSA DRUMMOND *** \\ VANESSA PAIVA FRANCO *

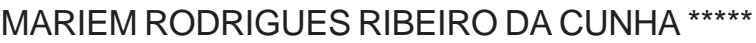 \\ HELOÍZA MARIA OLIVEIRA HORTA FRANKLIN * \\ TÂNIA MARAAMÂNCIO GUERRA PEIXOTO ***
}

\begin{abstract}
Amostras de polpas de morango industrializadas, comercializadas no estado de Minas Gerais, foram coletadas no período de 2006 a 2007 pelas Vigilâncias Sanitárias e encaminhadas à Fundação Ezequiel Dias para análise de resíduos de agrotóxicos. Realizou-se a extração pelo método de multirresíduos com acetona, diclorometano e hexano. Os extratos obtidos foram submetidos à análise por cromatografia a gás com detectores de captura de elétrons (CG-DCE), fotométrico de chama (CG-DFC) e nitrogênio fósforo (CG-DNF), espectrometria de massa (CG-MS) e cromatografia a líquido de alta eficiência (CLAE) com detectores ultravioleta (UV) visível e fluorescência. Os resultados evidenciaram o uso inadequado de agrotóxicos, sendo detectados produtos não autorizados como acefato, captana, clorfenapir, clorpirifós, dimetoato, ometoato, endossulfam, fenarimol, folpete, metamidofós, procloraz e tetradifona e os autorizados azoxistrobina, difenoconazol, fenpropatrina, iprodiona e procimidona. Isso indica que as boas práticas agrícolas não estão sendo cumpridas por alguns produtores e mostra a necessidade de fiscalização de alimentos processados, o que levaria à oferta de produtos de melhor qualidade.
\end{abstract}

PALAVRAS-CHAVE: AGROTÓXICOS; RESÍDUOS DE AGROTÓXICOS; POLPA DE MORANGO; CROMATOGRAFIA.

* Farmacêuticas, Serviço de Química, Fundação Ezequiel Dias, Belo Horizonte, MG (e-mail: angelicasissi@yahoo.com.br;vanessafranco@gmail.com;hfranklin@funed.mg.gov.br).

** Engenheira de Alimentos, Mestre em Tecnologia de Alimentos, Serviço de Química, Fundação Ezequiel Dias, Belo Horizonte, MG (e-mail: barbara.dias@funed.mg.gov.br).

*** Farmacêuticas, Mestres em Ciência de Alimentos, Serviço de Química, Fundação Ezequiel Dias, Belo horizonte,MG (e-mail: marcia.marcos@funed.mg.gov.br; aneliselapertosa@gmail.com; tania@ funed.mg.gov.br).

*** Química, Mestre em Química, Serviço de Química, Fundação Ezequiel Dias, Belo Horizonte, MG (e-mail: virna_quimica@yahoo.com.br).

*****Bóloga, Doutoranda em Ciência de Alimentos, Serviço de Química, Fundação Ezequiel Dias, Belo Horizonte, MG (e-mail: mariem@funed.mg.gov.br). 


\section{INTRODUÇÃO}

$\mathrm{Na}$ agricultura, grandes quantidades de agrotóxicos são utilizadas para o combate e a prevenção de pragas agrícolas. Desse modo, grande benefício para a produção agrícola é alcançado, garantindo alimento suficiente para a população (KRISTENSON et al., 2001). Apesar de sua importância econômica, os agrotóxicos são potencialmente tóxicos ao homem e seus resíduos são encontrados nos alimentos, na água e no meio ambiente (CALDAS e SOUZA, 2000; PRESIBELLA, 2004). Isso exige acompanhamento e quantificação dos produtos, como pressuposto para a rastreabilidade e proteção da saúde do homem e do meio ambiente (AMARAL, 2007).

O Brasil tem grande parte da sua economia baseada na atividade agrícola e foi classificado, em 2006, como o segundo maior consumidor de agrotóxicos do mundo e o oitavo em uso por área cultivada (ANVISA, 2006). É responsável, também, por 84\% do mercado de agrotóxicos da América Latina, cujo faturamento anual chega a US $\$ 5$ bilhões (BRASIL, 2008a).

A cultura de morango tem apresentado contaminação com resíduos de agrotóxicos acima do limite máximo permitido pela legislação e princípios ativos não autorizados, conforme observado nos programas de monitoramento realizados no Brasil (AMARAL e ATOÉ, 2005).

Minas Gerais é o maior produtor nacional de morango (CEAGESP, 2006), alcançando 40 mil toneladas por ano (o equivalente a $40 \%$ da produção nacional), seguido por São Paulo com 29 mil toneladas/ano e depois, respectivamente, por Rio Grande do Sul, Paraná e Santa Catarina.

GORENSTEIN (2000) estudou a presença de resíduos de agrotóxicos em alimentos frescos e observou que as amostras de morango analisadas apresentaram os maiores índices de resíduos de agrotóxicos em relação às outras frutas e vegetais. Entre os agrotóxicos encontrados nessa cultura, estavam o endossulfam, clorotalonil, dimetoato, metamidofós, vinclozalina, tetradifona, ometoato, acefato, monocrotofós e quintozeno.

O Decreto $n^{\circ} 4074$ de 04 de janeiro de 2002 (BRASIL, 2002), que regulamenta a Lei n 7.802/89 do Ministério da Agricultura, Pecuária e Abastecimento, instituiu que os agrotóxicos, seus componentes e afins só poderão ser produzidos, manipulados, importados, exportados, comercializados e utilizados no território nacional se previamente registrados em órgão federal competente, atendidas as diretrizes e exigências dos órgãos federais responsáveis pelos setores da saúde, do meio ambiente e da agricultura.

O presente trabalho teve como objetivo avaliar o grau de contaminação por agrotóxicos em polpas de morango industrializadas, coletadas em diversos municípios pelas Vigilâncias Sanitárias de Minas Gerais, nos anos de 2006 e 2007, visando à oferta de produtos de melhor qualidade.

\section{MATERIAL E MÉTODOS}

As Vigilâncias Sanitárias de Minas Gerais coletaram, no comércio de diversos municípios, 55 amostras de polpa de morango industrializadas e as encaminharam ao Laboratório de Resíduos de Pesticidas da Fundação Ezequiel Dias para análise.

Efetuou-se a análise dos agrotóxicos por meio de método de multirresíduos, conforme NETHERLANDS (1996a) e NETHERLANDS (1996b), mediante extração com acetona, seguida de partição com diclorometano e hexano, obtendo-se o extrato orgânico (Figura 1). Foram usados solventes e reagentes dos graus pesticida e CLAE. A partir do extrato obtido, alíquotas foram 
retiradas e preparadas de acordo com a técnica cromatográfica a ser utilizada na identificação e quantificação de 96 princípios ativos (relacionados nos resultados), sendo 12 isômeros e metabólitos. Foram utilizados os seguintes equipamentos para a realização das análises:

- Cromatógrafo a gás HP 5890 com detector fotométrico de chama (DFC) para análise de organofosforados, coluna HP5 (5\%-Phenyl-methylpolysiloxane metil), nitrogênio ultrapuro como gás de arraste e vazão constante de $10 \mathrm{~mL} / \mathrm{min}$. A programação de temperatura do forno foi de $60^{\circ} \mathrm{C}$ por $2 \mathrm{~min}, 30^{\circ} \mathrm{C} / \mathrm{min}$ até $170^{\circ} \mathrm{C}$ por $7 \mathrm{~min}, 20^{\circ} \mathrm{C} / \mathrm{min}$ até $215^{\circ} \mathrm{C}$ por $10 \mathrm{~min}$ e $30^{\circ} \mathrm{C} / \mathrm{min}$ até $280^{\circ} \mathrm{C}$ por $8 \mathrm{~min}$. O volume de injeção foi $2 \mu \mathrm{L}$.

\section{FIGURA 1 - FLUXOGRAMA DO MÉTODO DE MULTIRRESÍDUOS EM FRUTAS E HORTALIÇAS}

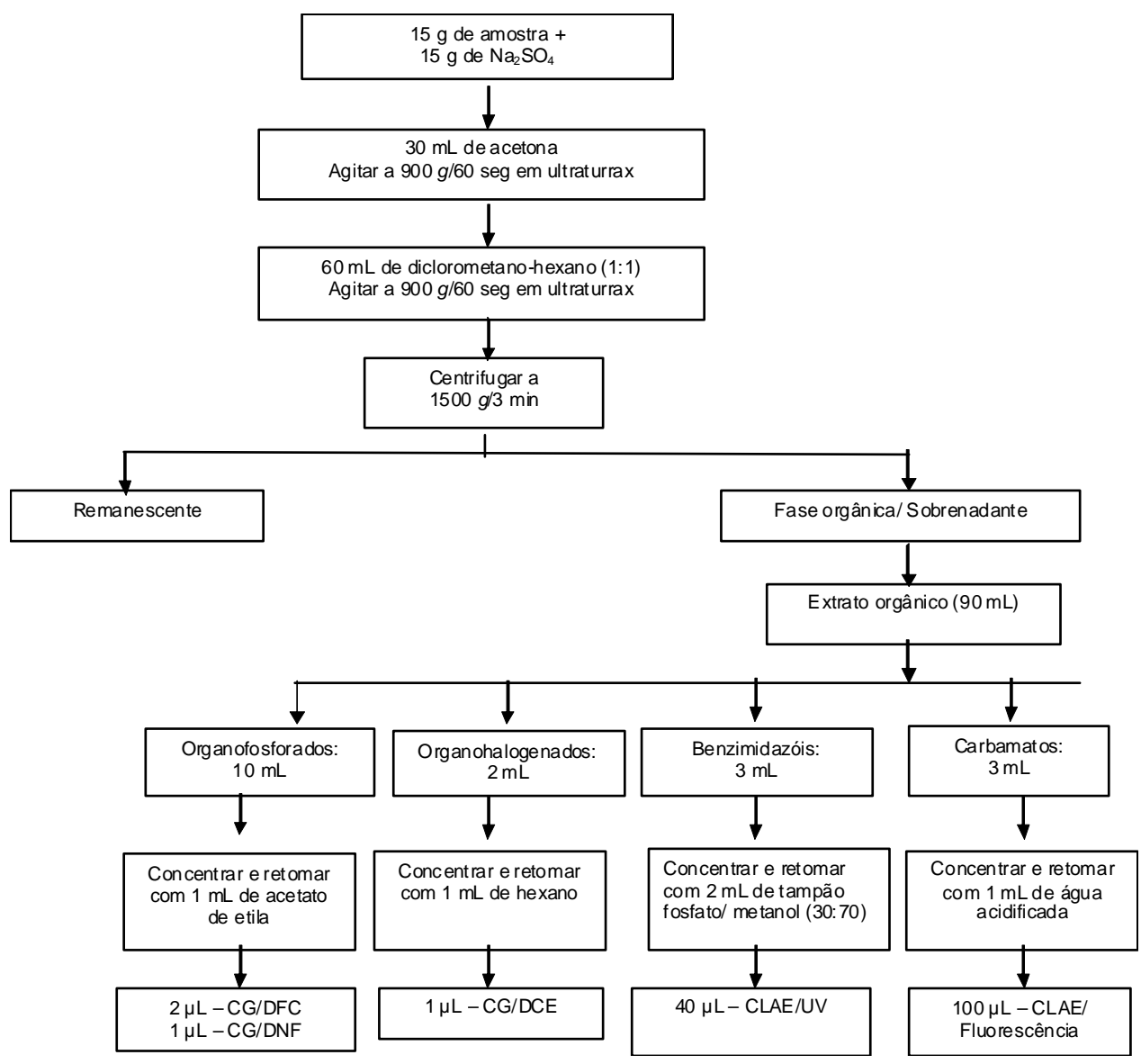

- Cromatógrafo a gás Agilent Technologies 6890 com detector nitrogênio fósforo (DNF) para análise de compostos nitrogenados e fosforados, coluna DB1701, nitrogênio ultrapuro como gás de arraste, com vazão constante de $1,4 \mathrm{~mL} / \mathrm{min}$. A programação de temperatura do forno foi $100^{\circ} \mathrm{C}$ por $2 \mathrm{~min}, 30^{\circ} \mathrm{C} / \mathrm{min}$ até $170^{\circ} \mathrm{C}$ por $6 \mathrm{~min}, 20^{\circ} \mathrm{C} / \mathrm{min}$ até $200^{\circ} \mathrm{C}$ por $8 \mathrm{~min}$ e $30^{\circ} \mathrm{C} / \mathrm{min}$ até $280^{\circ} \mathrm{C}$ por $14 \mathrm{~min}$. O volume de injeção foi $1 \mu \mathrm{L}$.

- Cromatógrafo a gás Agilent Technologies 6890 com dois detectores de captura de elétrons (DCE) e duas colunas distintas para análise de organoclorados e outros halogenados, colunas DB1701 (14\%-cyanopropyl-phenyl-methylpolysiloxane) e HP5 (5\%-Phenyl-methylpolysiloxane metil), nitrogênio ultrapuro como gás de arraste e vazão constante de $1,2 \mathrm{~mL} / \mathrm{min}$. A programação de temperatura do forno foi de $70^{\circ} \mathrm{C}$ por $1 \mathrm{~min}, 25^{\circ} \mathrm{C} / \mathrm{min}$ até $180^{\circ} \mathrm{C}$ por $2 \mathrm{~min}, 10^{\circ} \mathrm{C} / \mathrm{min}$ até $250^{\circ} \mathrm{C}$ por $10 \mathrm{~min} \mathrm{e} 15^{\circ} \mathrm{C} / \mathrm{min}$ até $280^{\circ} \mathrm{C}$ por $20 \mathrm{~min}$. O volume de injeção foi $1 \mu \mathrm{L}$. 
- Cromatógrafo a gás Agilent Technologies 6890 acoplado a espectrômetro de massas (CG-MS), utilizado para confirmação dos resultados, coluna HP5-MS (5\%-Phenyl-methylpolysiloxane metil) e hélio ultrapuro como gás de arraste. A programação de temperatura do forno foi de $100^{\circ} \mathrm{C}$ por $1 \mathrm{~min}, 20^{\circ} \mathrm{C} / \mathrm{min}$ até $150^{\circ} \mathrm{C}$, $10^{\circ} \mathrm{C} / \mathrm{min}$ até $250^{\circ} \mathrm{C}$ por 4 min e $30^{\circ} \mathrm{C} / \mathrm{min}$ até $280^{\circ} \mathrm{C}$ por $6,5 \mathrm{~min}$.

- Cromatógrafo a líquido de alta eficiência Shimadzu com detector ultravioleta visível para análise de benzimidazóis, coluna C18 $(4,6 \mathrm{~mm} \times 25 \mathrm{~cm}$, partícula de $5 \mu \mathrm{m})$, fase móvel composta por metanol: tampão fosfato $0,1 \% \mathrm{pH} 7,0$ (70:30), com vazão constante de $0,6 \mathrm{~mL} / \mathrm{min}$. A temperatura do forno foi de $30^{\circ} \mathrm{C}$ e o tempo de corrida de 14 minutos.

- Cromatógrafo a líquido de alta eficiência Waters com derivatização pós-coluna e detector de fluorescência para análise de carbamatos. Coluna Carbamatos Waters $(3,9 \mathrm{~mm} \times 15 \mathrm{~cm}$, partícula de $5 \mu \mathrm{m})$ com fase móvel composta por água ultrapura, metanol e acetonitrila em sistema de gradiente, com vazão constante de $1,5 \mathrm{~mL} / \mathrm{min}$. A temperatura do forno foi de $30^{\circ} \mathrm{C}$ e o tempo de corrida de 30 minutos.

Paralelamente, foram analisadas amostras testemunhas (branco) e realizados testes de recuperação, fortificando-se as amostras de polpa de morango com mistura de soluções padrões de analitos representativos dos agrotóxicos estudados.

Para garantir a correta execução do processo foi adicionada em todas as amostras, antes da extração, quantidade conhecida de padrão de clorpirifós metílico (surrogate). A recuperação desse padrão foi calculada em cada amostra e elaborada a carta controle.

\section{RESULTADOS E DISCUSSÃO}

\subsection{RECUPERAÇÕES DOS AGROTÓXICOS}

As análises das polpas fortificadas apresentaram resultados de $80 \%$ a 117\%, com coeficientes de variação (CV) na faixa de 5 a 20\% (Tabela 1). Os valores encontrados estão de acordo com o recomendado para análise de resíduos de agrotóxicos em alimentos, sendo aceitável a faixa de $70 \%$ a $120 \%$ de recuperação e coeficiente de variação inferior a 20\% (EUROPEAN COMISSION, 2007).

\section{TABELA 1 - RECUPERAÇÃO DOS AGROTÓXICOS EM POLPAS DE MORANGO}

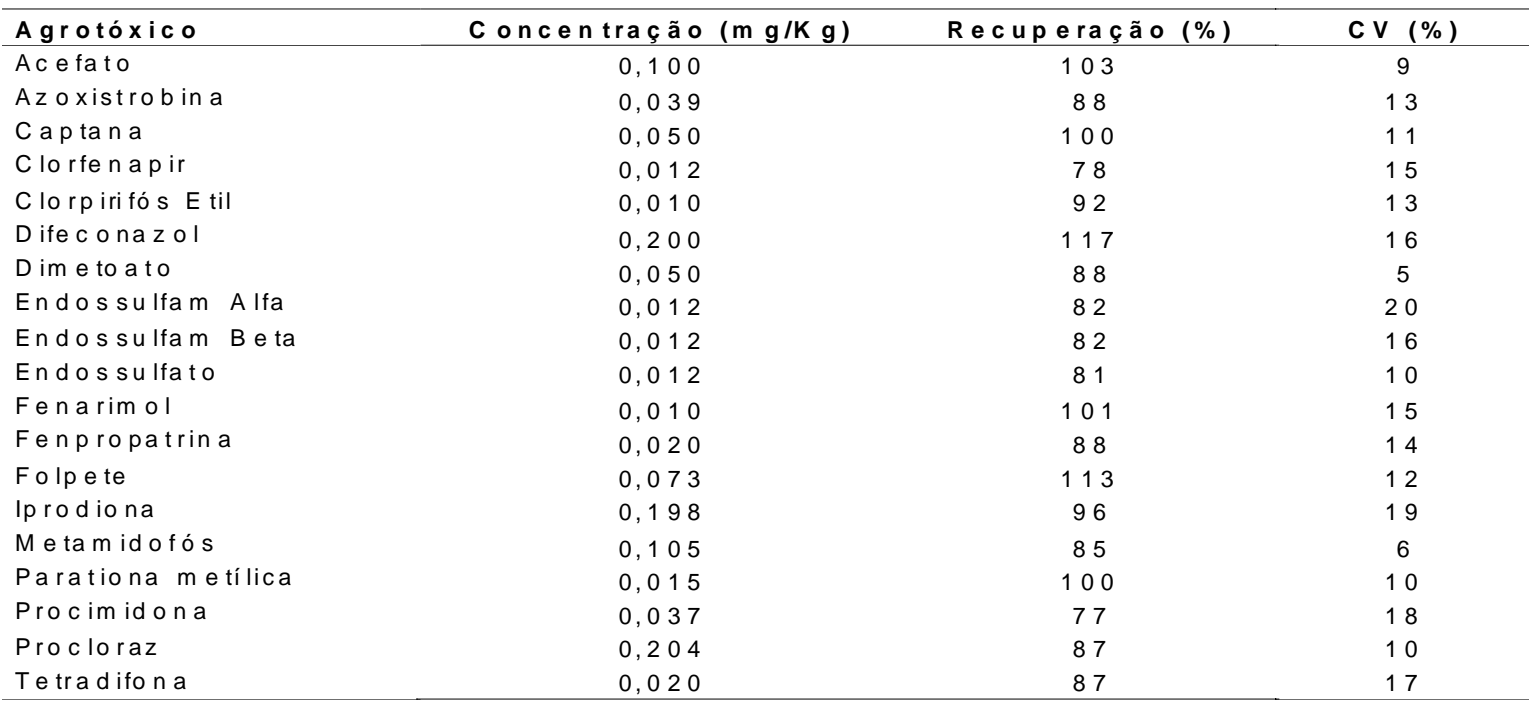

$\mathrm{CV}=$ coeficiente de variação. 


\subsection{NÍVEIS DE AGROTÓXICOS EM POLPAS DE MORANGO}

Das 55 amostras de polpa de morango industrializadas analisadas, 52 (95\%) revelaram resíduos de agrotóxicos. Entre essas, 27 (49\%) apresentaram produtos não autorizados como o acefato, captana, clorfenapir, clorpirifós, dimetoato, endossulfam, fenarimol, folpete, metamidofós, parationa metílica, procloraz e tetradifona (Figura 2).

\section{FIGURA 2 - RESULTADO DAS ANÁLISES DE POLPA DE MORANGO}

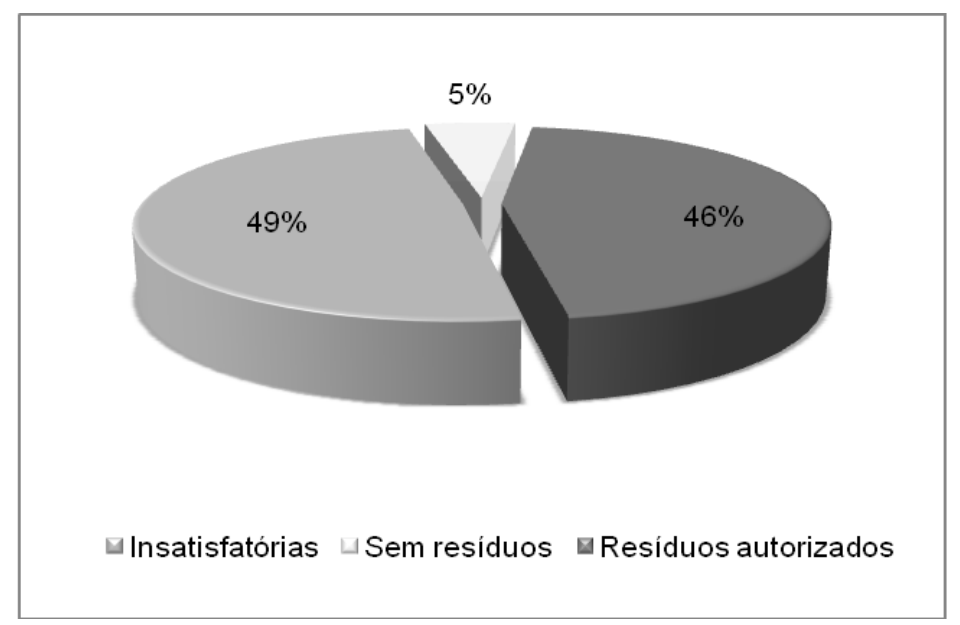

Trinta e cinco amostras (64\%) continham quatro ou mais princípios ativos, encontrando-se até dez tipos de resíduos na mesma amostra, dos quais seis não autorizados.

Azoxistrobina, fenpropatrina e procimidona foram os agrotóxicos autorizados para a cultura de morango que apareceram com maior frequência, 62\%, 82\% e 93\%, respectivamente. Foram detectados, também, resíduos de iprodiona e difenoconazol, porém todos com valores de acordo com os limites máximos de resíduos permitidos pela legislação vigente.

A Tabela 2 apresenta os agrotóxicos pesquisados, a faixa de concentração, os limites de detecção e de quantificação do método, bem como o limite máximo de resíduo permitido para a cultura de morango definido pela ANVISA (BRASIL,2008b) e os resultados dos resíduos encontrados nas amostras.

Os valores de dimetoato incluem o metabólito ometoato, conforme determina a Monografia de Produtos Agrotóxicos da Anvisa (BRASIL, 2008b). O resultado do endossulfam se refere à soma dos isômeros $\alpha, \beta$ e do metabólito endossulfato.

Dos 84 princípios ativos analisados, 17 estavam presentes como resíduos nas amostras de polpas de morango. Desses, 12 (70,6\%) não são autorizados para a cultura de morango. Esse resultado é condizente com o encontrado por GEBARA, CISCATO e FERREIRA (1999), que analisaram por cromatografia a gás 123 amostras de morango comercializadas na cidade de São Paulo. Foram pesquisados 68 princípios ativos e os resultados indicaram a presença de resíduos de pesticidas em $57,7 \%$ das amostras, sendo $39,0 \%$ de produtos não autorizados pela legislação vigente como o clorotalonil, dimetoato, endossulfato, metamidofós, quintozeno e tetradifona. 
TABELA 2 - LIMITES E RESÍDUOS DOS AGROTÓXICOS ESTUDADOS NAS AMOSTRAS DE POLPAS DE MORANGO

\begin{tabular}{|c|c|c|c|c|c|}
\hline AGROTÓXICOS & LD (m g/Kg) & LQ (mg/Kg) & $\begin{array}{c}\text { LMR } \\
(\mathrm{mg} / \mathrm{Kg})\end{array}$ & $\begin{array}{c}\text { Amostras com } \\
\text { resíduos (\%) }\end{array}$ & Níve is encontrados $(\mathrm{mg} / \mathrm{Kg})$ \\
\hline Acefato & 0,01 & 0,1 & $\mathrm{NA}$ & 12,7 & $<0,1$ a 0,21 \\
\hline Ald rin & 0,005 & 0,01 & NA & -- & - \\
\hline Aletrina & 0,03 & 0,05 & NA & --- & --- \\
\hline Azinfós é́lic o & 0,06 & 0,20 & NA & -- & -- \\
\hline Azinfós meálico & 0,05 & 0,10 & NA & --- & --- \\
\hline Azoxistrobina & 0,02 & 0,04 & 5 & 61,8 & $<0,07 \mathrm{a} 0,46$ \\
\hline Bifentrina & 0,02 & 0,06 & NA & --- & --- \\
\hline Bio aletrina & 0,03 & 0,05 & NA & --- & --- \\
\hline Bromoprop ilato & 0,01 & 0,02 & $\mathrm{NA}$ & --- & --- \\
\hline Captana & 0,03 & 0,05 & NA & 3,6 & $<0,05$ a 0,13 \\
\hline Carbaril & 0,04 & 0,10 & NA & -- & --. \\
\hline Carbendazim & 0,20 & 0,40 & 5,0 & --- & -.- \\
\hline Carbofenotiona & 0,01 & 0,03 & NA & --- & 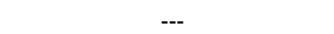 \\
\hline Carbofurano & 0,04 & 0,1 & NA & --- & --- \\
\hline Ciflutrina & 0,015 & 0,02 & NA & --- & --- \\
\hline Cipermetrina & 0.05 & 0,10 & NA & -.- & -.- \\
\hline Ciproconazol & 0,20 & 0,40 & NA & --- & --- \\
\hline Clordano & 0,01 & 0,02 & NA & --- & --- \\
\hline Clorfenvinfós & 0,02 & 0,06 & $\mathrm{NA}$ & --- & -- \\
\hline Clorfenapir & 0,005 & 0,01 & NA & 3,6 & $<0,01$ a 0,03 \\
\hline Clorotalonil & 0,02 & 0,06 & NA & --- & -.- \\
\hline Clorpirifós etil & 0,005 & 0,01 & NA & 2,0 & $<0,01$ \\
\hline Clorpirifós metílico & 0,01 & 0,02 & NA & -- & --- \\
\hline DDT-Total & 0,02 & 0,05 & NA & --- & --- \\
\hline Deltametrina & 0.03 & 0,05 & NA & -.- & -.- \\
\hline Diazinona & 0,005 & 0,01 & NA & --- & --- \\
\hline Diclorvós & 0,01 & 0,02 & NA & --- & --- \\
\hline Dicofol & 0,03 & 0,05 & NA & --- & --- \\
\hline $\begin{array}{l}\text { Dieldrin } \\
\text { Difenoconazol }\end{array}$ & 0,01 & 0,02 & NA & --- & --- \\
\hline Difenoconazol & 0,10 & 0,20 & 0,5 & 16,4 & $<0,20$ \\
\hline Dimeto ato & 0,02 & 0,05 & NA & 14,5 & $<0,05$ a 0,31 \\
\hline Dissulfotom & 0,01 & 0,02 & NA & -- & -- \\
\hline End ossulfam & 0,02 & 0,04 & NA & 3,6 & $<0,04$ \\
\hline End rim & 0,03 & 0,07 & NA & --- & --- \\
\hline Esfenvarel ato & 0,03 & 0,06 & NA & --- & -- \\
\hline Etiona & 0,005 & 0,01 & NA & --. & -.- \\
\hline Etoprofós & 0,02 & 0,05 & NA & --- & --- \\
\hline Etrinfós & 0,02 & 0,05 & NA & --- & --- \\
\hline Fenamifós & 0,01 & 0,02 & NA & --- & --- \\
\hline Fenarimol & 0,005 & 0,01 & NA & 2,0 & $<0,01$ \\
\hline Fenitrotiona & 0,005 & 0,01 & NA & --- & --. \\
\hline Fenprop atrin a & 0,01 & 0,02 & 2 & 82 & $<0,02$ a 0,27 \\
\hline Fentiona & 0,005 & 0,01 & NA & --- & -.- \\
\hline Fentoato & 0,005 & 0,01 & NA & --- & --- \\
\hline Fenvarel ato & 0,06 & 0,24 & NA & --- & --- \\
\hline Folpete & 0,04 & 0,07 & NA & 2,0 & $<0,04$ \\
\hline Forato & 0,005 & 0,01 & NA & -- & -.- \\
\hline $\mathrm{HCB}$ & 0,005 & 0,01 & NA & --- & --- \\
\hline $\mathrm{HCH}$ & 0,02 & 0,06 & NA & --- & --- \\
\hline Heptacloro & 0,005 & 0,01 & NA & --- & --- \\
\hline Heptacloro-epóxido & 0,005 & 0,01 & NA & --- & --- \\
\hline Imazalil & 0.03 & 0,05 & NA & -.. & -.. \\
\hline Iprodiona & 0,10 & 0,20 & 2 & 56,4 & $<0,2$ a 1,4 \\
\hline Lambd a-cial otrin a & 0,02 & 0,04 & NA & -- & --- \\
\hline Lindano & 0,01 & 0,02 & NA & --. & --. \\
\hline Mal ationa & 0,02 & 0,05 & 1,0 & --- & --- \\
\hline Metamidofós & 0,01 & 0,10 & NA & 14,5 & $? 0,10$ \\
\hline Metidationa & 0,01 & 0,02 & NA & -.- & 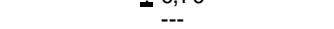 \\
\hline Metomil & 0,04 & 0,10 & NA & --- & --- \\
\hline Metoxicloro & 0,05 & 0,10 & NA & --- & --- \\
\hline Mevin fós & 0,03 & 0,10 & 1,0 & --- & --- \\
\hline Micl obutanil & 0,05 & 0,10 & NA & --. & -.- \\
\hline Mirex & 0,03 & 0,07 & NA & --- & --- \\
\hline Monocrotofós & 0,01 & 0,02 & NA & --- & -- \\
\hline Oxifluorfem & 0,005 & 0,01 & NA & --- & --- \\
\hline Paration a etílica & 0,01 & 0,02 & NA & --- & --- \\
\hline Paration ametílica & 0,005 & 0,01 & NA & 2,0 & 0,02 \\
\hline Permetrina & 0,03 & 0,07 & NA & -- & --- \\
\hline Pirazofós & 0,02 & 0,04 & NA & --- & --- \\
\hline Pirimifós et liico & 0,02 & 0,05 & NA & --- & --- \\
\hline Pirimifós metílic o & 0,005 & 0,01 & NA & --- & --- \\
\hline Procimid ona & 0,02 & 0,04 & 3 & 93 & $<0,04$ a 0,55 \\
\hline Proclor az & 0,10 & 0,20 & NA & 5,4 & $? 0,20$ \\
\hline Profenofós & 0,02 & 0,04 & 3,0 & --- & --- \\
\hline Propiconazol & 0,05 & 0,20 & NA & --- & --- \\
\hline Protiofós & 0,005 & 0,01 & NA & '-- & --- \\
\hline Tebuconazol & 0.05 & 0,10 & 0,1 & --. & --. \\
\hline Terbufos & 0,01 & 0,03 & NA & --- & --- \\
\hline Tetradifona & 0,005 & 0,02 & NA & 29 & ? 0,03 \\
\hline Tiaben dazol & 0,25 & 0,50 & NA & -- & -.- \\
\hline Triazofós & 0,03 & 0,06 & NA & --- & --- \\
\hline Triclorfom & 0,07 & 0,10 & NA & --- & --- \\
\hline Trifluralina & 0,02 & 0,05 & NA & --- & -- \\
\hline
\end{tabular}

LD = limite de detecção, LQ = limite de quantificação, LMR = limite máximo de resíduo, NA = não autorizado. 


\section{CONCLUSÃO}

A avaliação do número de agrotóxicos utilizados na mesma cultura evidenciou que, muitas vezes, esse número é excessivo e que vários produtos são utilizados de maneira irregular.

Este estudo mostrou que a preocupação com os vegetais in natura deve ser estendida aos produtos processados a partir do fruto, já que a contaminação verificada nas polpas de morango foi significativa. A fiscalização de produtos processados pode contribuir para a oferta de produtos com melhor qualidade, uma vez que a indústria vai se preocupar em adquirir matéria-prima mais pura, induzindo, consequentemente, o agricultor a utilizar corretamente os agrotóxicos e apenas quando necessário.

\section{ABSTRACT}

\section{EVALUATION OF PESTICIDES RESIDUES IN INDUSTRIALIZED STRAWBERRY PULPS}

Samples of industrialized strawberry pulps, commercialized in Minas Gerais state, Brazil, were collected in the period from 2006 to 2007 by the Sanitary Surveillances, and were sent to Fundação Ezequiel Dias for analysis of pesticide residues. It was performed the extraction by multi-residue method with acetone, dichloromethane and hexane. The obtained extracts were analyzed by gas chromatography with detectors of electron capture (GC-ECD), flame photometric (GC-FPD), nitrogen phosphorus (GC-NPD), mass spectrometry (GC-MS) and high performance liquid chromatography (HPLC) with UV visible and fluorescence detectors. The results showed the inappropriate use of pesticides, which are not authorized as acephate, captan, chlorfenapyr, chlorpyrifos, dimethoate, omethoate, endosulfan, fenarimol, folpet, methamidophos, prochloraz and tetradifon, and the permitted ones azoxystrobin, difenoconazole, fenpropatrina, iprodione and procymidone. These indicate that good agricultural practices are not being followed by some producers, and demonstrate the necessity of processed food fiscalization, which will lead to products of better quality.

KEY-WORDS: PESTICIDES; RESIDUES; STRAWBERRY PULP; CHROMATOGRAPHY.

\section{REFERÊNCIAS}

1 ANVISA. Agência Nacional de Vigilância Sanitária. Resíduos de agrotóxicos em alimentos. Revista de Saúde Pública, São Paulo, v. 40, n. 2, p. 361-363, 2006.

2 AMARAL, E.H.; ALTOÉ, I.M.F.F. Monitoramento de resíduos de agrotóxicos no morango de Minas Gerais. Boletim do morango: cultivo convencional, segurança alimentar, cultivo orgânico. Belo Horizonte: Ceasa Minas, 2005. p. 137-142.

3 BRASIL. Ministério da Agricultura, Pecuária e Abastecimento. Legislação federal de agrotóxicos e afins. Decreto $\mathbf{n}$. 4074 de 04 de janeiro de 2002. Disponível em: http://extranet.agricultura.gov.br/sislegis-consulta/ consultarLegislacao.do?operacao=visualizar\&id=16764. Acesso em: 30 jan. 2009.

4 BRASIL. Agência Nacional de Vigilância Sanitária. Brasil é referência na América Latina. 2008a. Disponível em: <http://www.anvisa.gov.br/divulga/noticias/2008/091208_link.htm>. Acesso em: 26 jan. 2009.

5 BRASIL. Agência Nacional de Vigilância Sanitária. Monografias de produtos agrotóxicos. 2008b. Disponível em: www.anvisa.gov.br/toxicologia/monografias/index.htm. Acesso em: 2 fev. 2009.

6 CALDAS, E.D.; SOUZA, L.C.K.R. Avaliação de risco crônico na ingestão de resíduos de pesticidas na dieta brasileira. Revista de Saúde Pública, São Paulo, v. 34, n. 5, p. 529-537, 2000.

7 CEAGESP. Sistema de Informação de Mercado da Companhia de Entrepostos e Armazéns Gerais de São Paulo. Morango. São Paulo, 2006.

8 EUROPEAN COMISSION. Document n., SANCO/2007/3131. Method validation and quality control. Procedures for pesticide residues analysis in food and feed. 2007. 38 p. Disponível em: <http://ec.europa.eu/food/plant/protection/ resources/qualcontrol_en.pdf> Acesso em: 13 agos. 2009.

9 FUTINO, A.M.; SILVEIRA, J.M.J.F. da. A indústria de defensivos agrícolas no Brasil. Agricultura em São Paulo, São Paulo, v. 38, p. 1-44, 1991. 
10 GEBARA, A.B.; CISCATO, C.H.P.; FERREIRA, M. da S. Resíduos de pesticidas em morangos comercializados na cidade de São Paulo, de 1994 a 1996. Higiene Alimentar, São Paulo, v. 13, n. 66/67, p. 100-103, nov./dez. 1999.

11 GORENSTEIN, O. Uma abordagem sobre resíduos de agrotóxicos em alimentos frescos. Informações Econômicas, São Paulo, 2000. Disponível em: <http:www.iea.sp.gov.br/out/verTexto.php?codTexto=520>. Acesso em: 26 jan. 2009.

12 KRISTENSON, E.M.; HAVERKATE, E.G.J.; SLOOTEN, C.J.; RAMOS, L.; VREULS, R.J.J.; BRINKMAN, U. A. Miniaturized automated matrix solid-phase dispersion extraction of pesticides in fruit followed by gas chromatographic-mass spectrometric analysis. Journal of Chromatography, v. 917, p. 277-286, 2001.

13 NETHERLANDS. Ministry of Public Health, Welfare and Sport. Method 1: multi-residue pesticides amenable to gas chromatography. In: ANALYTICAL methods for pesticide residues in foodstuffs. $6^{\text {th }}$ ed. Amsterdam: RIVM, 1996a.

14 NETHERLANDS. Ministry of Public Health, Welfare and Sport. Method 2: Multi-residue pesticides analyzed with HPLC procedures. In: ANALYTICAL methods for pesticide residues in foodstuffs. $6^{\text {th }}$ ed. Amsterdam: RIVM, 1996b.

15 PRESIBELLA, K.M. Efeitos da associação dos pesticidas deltametrina e endosulfano sobre o sistema reprodutivo de ratos wistar. Curitiba, 2004, 125 f. Dissertação (Mestrado em Farmacologia) - Setor de Ciências Biológicas, Universidade Federal do Paraná.

\section{AGRADECIMENTOS}

Às Vigilâncias Sanitárias de Minas Gerais pela contribuição. 